\title{
Consistency of Knee Pain and Risk of Knee Replacement: The Multicenter Osteoarthritis Study
}

\author{
BARTON L. WISE, DAVID T. FELSON, MARGARET CLANCY, JINGBO NIU, TUHINA NEOGI, NANCY E. LANE, \\ JEAN HIETPAS, JEFFREY R. CURTIS, LAURENCE A. BRADLEY, JAMES C. TORNER, and YUQING ZHANG
}

\begin{abstract}
Objective. To examine whether the consistency or persistence of knee pain, in addition to its severity, predicts incident total knee replacement (TKR).

Methods. The Multicenter Osteoarthritis Study (MOST) is a longitudinal study of persons aged 50 to 79 years with symptomatic knee osteoarthritis or at high risk of disease. Subjects were queried about the presence of knee pain on most days of the previous 30 days (i.e., frequent knee pain; FKP) at 2 timepoints: a telephone screen followed by a clinic visit (median separation 4 weeks). We defined a knee as having "consistent pain" if the subject answered positively to the FKP question at both timepoints, "inconsistent pain" if FKP was positive at only one timepoint, or as "no FKP" if negative at both. We examined the association between consistent FKP and risk of TKR using multiple binomial regression with generalized estimating equations.

Results. In 3026 persons (mean age 63 yrs, mean body mass index 30.4), 2979 knees (50\%) had no FKP at baseline, 1279 knees (21.5\%) had inconsistent FKP, and 1696 knees (28.5\%) had consistent FKP. Risk of TKR over 30 months was $0.8 \%, 2.6 \%$, and $8.8 \%$ for knees with no, inconsistent, and consistent FKP, respectively. Relative risks of TKR over 30 months were 1.2 (95\% CI 0.6-2.3) and 2.3 (95\% CI 1.2-4.4) for knees with inconsistent and consistent FKP, compared with those without FKP. This association was consistent across each level of pain severity on the Western Ontario and McMaster Universities Osteoarthritis Index.

Conclusion. Consistency of frequent knee pain is associated with an increased risk of TKR independently of knee pain severity. (First Release April 15 2011; J Rheumatol 2011;38:1390-5; doi:10.3899/jrheum.100743)
\end{abstract}

Key Indexing Terms: OSTEOARTHRITIS

PAIN

Osteoarthritis (OA) is the most common form of arthritis, which afflicts 27 million US adults, almost 6 million more than in $1995^{1}$. Although estimates for prevalence vary in different studies, the National Health and Nutrition Examination Survey III found that $12 \%$ of US adults 60 years of age or older experience symptomatic knee $\mathrm{OA}^{2}$, defined as pain on most days with radiological OA. It is

From the Center for Healthy Aging, University of California, Davis School of Medicine, Sacramento, California; and the Clinical Epidemiology Research and Training Unit, Boston University School of Medicine, Boston, Massachusetts, USA.

The Multicenter Osteoarthritis Study is supported by National Institutes of Health grants UO1 AG18820 (Dr. Felson); UO1 AG18832 (Dr. Torner); UO1 AG18947 (Dr. Lewis); and UO1 AG19069 (Dr. Nevitt). Dr. Wise was supported by UC Davis Building Interdisciplinary Research Careers in Women's Health Program grant NIH K12HD051958.

B.L. Wise, MD, MSc, Center for Healthy Aging, UC Davis, Boston University School of Medicine; D.T. Felson, MD, MPH; M. Clancy, MS, MPH; J. Niu, DSc; T. Neogi, MD, PhD, Boston University School of Medicine; N.E. Lane, MD, Center for Healthy Aging, UC Davis, J. Hietpas, LCSW, OTR, University of California, San Francisco, San Francisco, CA; J.R. Curtis, MD, MPH; L.A. Bradley, PhD, University of Alabama, Birmingham, AL; J.C. Torner, MS, PhD, University of Iowa, Iowa City, IA; Y. Zhang, DSc, Boston University School of Medicine. Address correspondence to Dr. B.L. Wise, Center for Healthy Aging, University of California, Davis Medical Center, 4800 2nd Avenue, Suite 2600, Sacramento, CA 95817.E-mail: barton.wise@ucdmc.ucdavis.edu Accepted for publication October 29, 2010.

\section{JOINT REPLACEMENT KNEE}

expected that with the increase in average body mass index (BMI) of the US population over the last several decades $3,4,5$ and the established association between high BMI and knee $\mathrm{OA}$, the prevalence of knee OA will increase in the population over the next several decades. This increase will be exacerbated by the simultaneous aging of the US population.

Total knee replacement (TKR) is an effective therapy to control pain and improve function in subjects with advanced knee OA. TKR is usually recommended for those with the most severe OA. This treatment has become steadily more common over the last 2 decades, with a $53 \%$ increase in TKR from the year 2000 to year $2004^{6}$ and a $70 \%$ increase from 1996 to $2006^{7}$. These trends are projected to continue well into the future. However, the characteristics of patients' symptoms that influence who will have TKR are poorly understood. For most patients with knee OA, pain fluctuates in intensity. The Boston Osteoarthritis Study found that 39\% of those with symptomatic radiographic knee OA had Western Ontario and McMaster Universities Osteoarthritis Index (WOMAC) pain score that varied from little or no pain to severe pain at 3 times over 3 years $^{8}$. The Longitudinal Examination of Arthritis Pain study found significant weekly variations in self-rated pain ${ }^{9}$.

Numerous studies have examined risk factors in relation Personal non-commercial use only. The Journal of Rheumatology Copyright @ C 2011. All rights reserved. 
to utilization of TKR, including age, sex, BMI, race, ethnicity, general health status, willingness to consider joint replacement, and regional differences, but few studies have assessed various measures of knee symptoms in relation to risk of $\mathrm{TKR}^{10,11}$. Hawker, et al found that worse baseline knee pain severity measured by WOMAC was associated with $\mathrm{TKR}^{12}$. Neogi, et al used consistency of pain in examining the association of measures of pain with radiographic knee $\mathrm{OA}^{13}$. However, to our knowledge, few if any studies have compared the influence of the dimensions of OA pain on risk of TKR. Our study examined the consistency or persistence of knee pain over time and its relation to risk of TKR.

\section{MATERIALS AND METHODS}

The Multicenter Osteoarthritis Study (MOST) is a NIH-funded observational study of persons aged 50 to 79 years either with knee OA or at high risk of development of knee OA, including overweight and obese persons and those with knee pain, aching, or stiffness during most of the previous 30 days or a history of knee injury or knee surgery. Details of the MOST study and study population have been published ${ }^{14}$. The MOST Study is in compliance with the Helsinki Declaration, and approval has been given by the institutional review boards at each participating site.

At baseline, subjects were queried about the presence of knee pain on most days, completed a knee-specific WOMAC, and obtained bilateral weight-bearing posteroanterior and lateral radiographs. Presence of knee pain, WOMAC score, and radiographic OA severity were determined again at the 30-month followup visit. Radiographs were read paired by 2 readers with an adjudication panel for disagreements.

Subjects were queried at baseline about the presence of knee pain on most days of the previous 30 days. We will refer to this as the frequent knee pain (FKP) question. This same question was asked twice: once at a telephone screening and a second time at the baseline clinic visit. These 2 timepoints were separated by a median time of 33 days. Other demographic and clinical information was also collected at the baseline clinic visit, and this information was used for adjustment in the analyses here. Data on incident TKR were collected at the 15-month and 30-month followup visits based on self-report, with confirmation by radiograph or medical documentation.

We defined a knee as having "consistent pain" if the subject answered positively to the FKP question at both timepoints, or as "inconsistent pain" if he/she answered the FKP question positively at only one of the 2 timepoints, or as "no FKP" if he/she answered negatively at both timepoints. We calculated the risk of TKR over 30 months for knees with no FKP, with inconsistent FKP, and with consistent knee pain. We examined the association between consistent FKP and risk of TKR using generalized estimating equations to account for the correlation between 2 knees within one subject. In the multiple regression model that we used to estimate relative risk we adjusted for age, sex, race, education, BMI, depression as measured by Center for Epidemiologic Studies Depression Scale (CES-D), clinical site, occupation, and radiographic Kellgren-Lawrence grade.

In addition, we divided knee pain severity, measured by a knee-specific WOMAC pain subscale, into 3 categories, mild (0-2), moderate $(>2-6)$, or severe (>6-20), and created a composite variable with 9 categories to represent both consistency and severity of pain (mild WOMAC pain without FKP, mild WOMAC pain with inconsistent FKP, mild WOMAC pain with consistent FKP, moderate WOMAC pain without FKP, moderate WOMAC pain with inconsistent FKP, moderate WOMAC pain with consistent FKP, severe WOMAC pain without FKP, severe WOMAC pain with inconsistent FKP, severe WOMAC pain with consistent FKP). We examined the association between the composite variable at baseline and risk of TKR using logistic regression. To assess whether the association between consistent FKP and risk of TKR was modified by other risk factors, we evaluated the effect of consistent knee pain across strata of each potential risk factor. To address the question of residual confounding by pain severity when examining the effect of consistent knee pain on TKR, besides the analyses described above, we also performed an analysis limited to subjects with WOMAC pain of 0 or 1 , who by definition did not answer any WOMAC pain severity subscale question with "moderate" or "severe" pain; this allows examination of the effect of consistency of pain in those with pain that is not revealed by WOMAC severity score. SAS 9.1.3 statistical software was used for these analyses.

\section{RESULTS}

Of the 3026 participants enrolled at baseline, 78 knees had TKR prior to their entry into the study, and these knees were excluded from the present analysis. Over the 30-month followup period, 202 of 5974 knees (3.4\%) had a TKR; of these 202 TKR, 190 (94\%) were confirmed by radiograph or medical documentation.

Table 1 shows the characteristics of subjects with and without TKR. Compared with those without TKR, subjects with TKR were more likely to be women (60\% vs $71 \%)$, older (62 vs 65 yrs old), have higher prevalence of ROA $(34.5 \%$ vs $93.6 \%)$, and have higher WOMAC pain scores (3.3 vs 7.3).

Table 2 shows the distribution of potential risk factors for TKR according to categories of FKP. At baseline 2979 knees (50\%) had no FKP, 1279 knees $(21.5 \%)$ had inconsistent FKP, and 1696 knees (28.5\%) had consistent FKP. As compared with those with no FKP, those with inconsistent and consistent pain were more likely to be female, have ROA, and have higher mean WOMAC pain.

Table 1. Characteristics of 3026 study participants in MOST study, according to presence or absence of incident total knee replacement (TKR); participant counted once for each knee they contribute.

\begin{tabular}{lcc}
\hline Characteristic & $\begin{array}{c}\text { No TKR, } \\
\mathrm{n}=5666\end{array}$ & $\begin{array}{c}\mathrm{TKR}, \\
\mathrm{n}=202\end{array}$ \\
\hline Age, mean yrs (SD) & $62.3(8.1)$ & $64.8(7.5)$ \\
Sex, & & \\
$\quad$ Male & 40 & 29.2 \\
$\quad$ Female & 60 & 70.8 \\
Radiographic osteoarthritis, Kellgren-Lawrence grade, $\%$ & \\
0 & 31.0 & 1.5 \\
1 & 34.5 & 5.0 \\
2 & 13.1 & 10.4 \\
3 & 13.7 & 29.7 \\
4 & 7.7 & 53.5 \\
WOMAC score, mean (SD) & $3.3(3.7)$ & $7.3(3.8)$ \\
Occupation, \% & & \\
Farming & 6.1 & 4.0 \\
Un/semiskilled & 6.0 & 6.4 \\
Worked at home & 7.7 & 7.4 \\
Other & 80.1 & 82.2 \\
Education, \% & & \\
High school or less & 28.5 & 37.6 \\
Some college & 26.6 & 28.2 \\
College graduate or higher & 44.8 & 34.2 \\
\hline
\end{tabular}

WOMAC: Western Ontario and McMaster Universities OA pain score. Personal non-commercial use only. The Journal of Rheumatology Copyright @ 2011. All rights reserved. 
Table 2. Baseline characteristics of study participants according to categories of frequent knee pain (FKP). Participants contributing 2 knees are counted twice.

\begin{tabular}{|c|c|c|c|}
\hline Characteristic & $\begin{array}{l}\text { No FKP, } \\
\mathrm{n}=2974\end{array}$ & $\begin{array}{l}\text { Inconsistent FKP, } \\
\mathrm{n}=1273\end{array}$ & $\begin{array}{c}\text { Consistent FKP, } \\
\mathrm{n}=1687\end{array}$ \\
\hline Age, mean yrs & 62.5 & 62.8 & 62.0 \\
\hline \multicolumn{4}{|l|}{ Sex $\%$} \\
\hline Male & 44.3 & 36.6 & 34.9 \\
\hline Female & 55.7 & 63.4 & 65.1 \\
\hline \multicolumn{4}{|l|}{ Race. \% } \\
\hline White & 86.9 & 81.3 & 78.5 \\
\hline Non-white & 13.2 & 18.7 & 21.5 \\
\hline Body mass index, mean & 30.1 & 30.6 & 31.8 \\
\hline \multicolumn{4}{|c|}{ Radiographic osteoarthritis, Kellgren-Lawrence grade, $\%$} \\
\hline 0 & 53.5 & 39.8 & 27.7 \\
\hline 1 & 21.2 & 19.2 & 14.3 \\
\hline 2 & 12.1 & 15.1 & 14.6 \\
\hline 3 & 11.4 & 18.7 & 25.4 \\
\hline 4 & 1.8 & 7.2 & 18.0 \\
\hline WOMAC, mean (range 0-20) & 1.2 & 3.9 & 7.1 \\
\hline \multicolumn{4}{|l|}{ Education, $\%$} \\
\hline High school or less & 26.1 & 30.6 & 33.0 \\
\hline Some college & 25.6 & 28.8 & 27.4 \\
\hline College graduate or higher & 48.4 & 40.6 & 39.6 \\
\hline \multicolumn{4}{|l|}{ Site, $\%$} \\
\hline Iowa & 55.1 & 50.3 & 40.1 \\
\hline Alabama & 44.9 & 49.7 & 59.9 \\
\hline \multicolumn{4}{|l|}{ Occupation, $\%$} \\
\hline Labor & 36.3 & 39.5 & 37.3 \\
\hline Not labor & 44.6 & 41.2 & 41.1 \\
\hline Other & 19.1 & 19.3 & 21.5 \\
\hline \multicolumn{4}{|l|}{ Depressive symptoms, $\%$} \\
\hline None & 89.0 & 82.7 & 76.9 \\
\hline Mild to moderate & 6.6 & 10.5 & 12.2 \\
\hline Possible major & 4.4 & 6.8 & 10.9 \\
\hline
\end{tabular}

WOMAC: Western Ontario and McMaster Universities OA pain score.

As shown in Table 3, of those knees with no frequent knee pain, $0.8 \%$ had a TKR, while $2.6 \%$ of those with inconsistent frequent pain and $8.8 \%$ of those with consistent pain had a TKR over the 30-month period. The adjusted relative risk for TKR for those with consistent pain as compared with those knees with no pain was 2.3 (95\% CI 1.2-4.4; $\mathrm{p}$ for trend $=0.0004$ ).

Adjusting for additional potential confounding variables did not affect the association. Even in an analysis stratified by WOMAC pain severity, consistency of pain was still associated with an increased risk of TKR (Figure 1, Table
4). Within each stratum of WOMAC pain, we observed increasing risk of TKR across the consistency categories, with the exception of persons with moderate WOMAC and no FKP, who did not fit the pattern. Those with both consistent FKP and a WOMAC score in the severe category had 8.0 times the risk of TKR compared with those with no FKP and mild WOMAC (95\% CI 3.9-16.1).

Considering that knees with consistent FKP are likely to have more severe knee pain, we performed an additional analysis of FKP and risk of TKR by limiting to knees with WOMAC pain equal to 0 or 1 (with a total of 13 incident

Table 3. Relation of consistence of pain to risk of total knee replacement (TKR). Adjusted for age, sex, race, education, body mass index, depressive symptoms measured by CES-D, clinical site, occupation, and baseline cross-sectional radiographic Kellgren-Lawrence grade.

\begin{tabular}{lcccc}
\hline $\begin{array}{l}\text { Consistency of frequent } \\
\text { knee pain (FKP) }\end{array}$ & Total No. Knees & No. with TKR & $\begin{array}{c}\text { Risk Over } \\
\text { 30 Months }\end{array}$ & $\begin{array}{c}\text { Adjusted Relative Risk } \\
\text { Over 30 Months (95\% CI) }\end{array}$ \\
\hline No FKP & 2937 & 24 & 0.8 & 1.0 \\
Inconsistent FKP & 1247 & 32 & 2.6 & $1.2(0.6-2.3)$ \\
Consistent FKP & 1669 & 146 & 8.8 & $2.3(1.2-4.4)$ \\
\hline
\end{tabular}




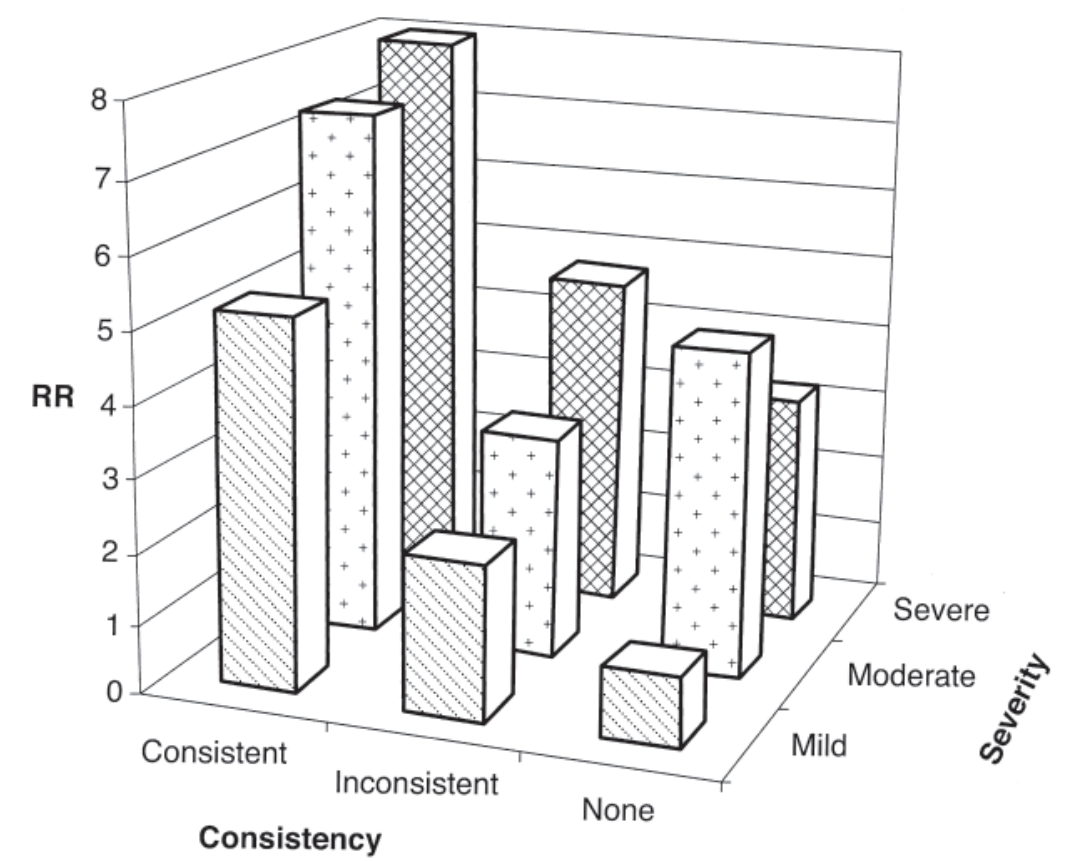

Figure 1. Adjusted relative risk (RR) of total knee replacement: severity of WOMAC pain versus consistency in a knee-based analysis. RR adjusted for age, sex, race, education, body mass index, depressive symptoms measured by CES-D, clinical site, occupation, and baseline cross-sectional radiographic Kellgren-Lawrence grade.

Table 4. Adjusted relative risk (RR) of total knee replacement (TKR): severity of Western Ontario and McMaster Universities pain score (WOMAC) pain versus consistency in a knee-based analysis. RR adjusted for age, sex, race, education, body mass index, depressive symptoms measured by CES-D, clinical site, occupation, and baseline cross-sectional radiographic Kellgren-Lawrence grade.

\begin{tabular}{|c|c|c|c|c|c|c|}
\hline \multirow[b]{2}{*}{ Severity of Pain, WOMAC } & \multicolumn{2}{|c|}{ No FKP } & \multicolumn{2}{|c|}{$\begin{array}{l}\text { Consistency of Pain } \\
\text { Inconsistent FKP }\end{array}$} & \multicolumn{2}{|c|}{ Consistent FKP } \\
\hline & $\begin{array}{l}\text { Portion with } \\
\text { TKR, \% }\end{array}$ & $\begin{array}{l}\text { Adj RR } \\
(95 \% \text { CI })\end{array}$ & $\begin{array}{l}\text { Portion with } \\
\text { TKR, \% }\end{array}$ & $\begin{array}{l}\text { Adj RR } \\
(95 \% \text { CI })\end{array}$ & $\begin{array}{l}\text { Portion with } \\
\text { TKR, \% }\end{array}$ & $\begin{array}{l}\text { Adj RR } \\
(95 \% \text { CI })\end{array}$ \\
\hline Mild (0-2) & 9/2410 (0.4) & 1.0 (referent) & $6 / 507(1.2)$ & $2.2(0.7-6.8)$ & 6/177 (3.4) & $5.2(1.8-15.2)$ \\
\hline Moderate (> 2-6) & $13 / 449(2.9)$ & $4.6(2.0-10.4)$ & $15 / 521(2.9)$ & $3.1(1.3-7.4)$ & $46 / 610(7.5)$ & $7.4(3.6-15.0)$ \\
\hline Severe $(>6-20)$ & 2/75 (2.7) & $3.2(0.7-14.4)$ & $11 / 218(5.1)$ & $4.7(2.0-10.7)$ & 94/881 (10.7) & $8.0(3.9-16.1)$ \\
\hline
\end{tabular}

FKP: frequent knee pain.

TKR). Compared with knees with no FKP, the relative risk of TKR was 3.9 (95\% CI 1.0-14.8) in those with inconsistent pain and $9.6(95 \%$ CI $2.5-37.1)$ in those with consistent pain. In a further analysis limited to knees with WOMAC pain equal to 0 (with a total of 10 incident TKR), compared with knees with no FKP, the relative risk of TKR was 4.7 (95\% CI 1.8-26.2) in those with inconsistent pain and 10.2 (95\% CI 1.7-69.1) in those with consistent pain.

Persons with consistent FKP had an increased risk of TKR over 30 months across all strata of other factors. No interaction term was statistically significant, including those related to sex, age, education, geographic location, and radiographic severity.

\section{DISCUSSION}

In this prospective cohort study, we found that consistency of frequent knee pain was strongly associated with incident TKR over a 30-month period. The association persisted even after control for pain severity and other potential confounders. In addition, such an association does not appear to be modified by sex or other factors.

Many studies have reported a significant increase in the number of TKR being performed on younger patients. Jain, et al reported that between 1990 and 2000, the proportion of TKR in persons $50-59$ years old increased by only $53.7 \%$, while the same proportion in the 40-49 year age group increased by a much larger $95.2 \%$. This trend was also noted more recently by Kim, who found that between 2000 and 2004, TKR increased in number by $38 \%$ in those aged 65-84 years, while those aged 45-64 experienced an increase in number of TKR of $83 \%{ }^{6}$. These trends may be due to greater confidence in the extended durability of Personal non-commercial use only. The Journal of Rheumatology Copyright @ 2011 . All rights reserved. 
newer prosthetic knees, and also possibly due to greater need for TKR in younger age groups and greater willingness to consider TKR by those same younger persons. It may help to rationalize these changes in practice to incorporate a strong predictor of ultimate TKR into replacement guidelines.

Several investigators have attempted to create guidelines to help identify candidates for TKR who might benefit from the procedure. Escobar, et al used the RAND appropriateness method, which combines expert opinion with clinical evidence, and created an explicit model that included age, location, function and symptoms, mobility, and stability as well as radiological measures, which they subsequently validated using health-related quality of life measurements ${ }^{16,17}$. De Coster, et al developed a priority referral score for TKR in Western Canada that included questions about pain with various activities, but that did not refer to frequency or consistency of pain ${ }^{18}$. In 2003, the National Institutes of Health released a consensus statement on guidelines for TKR that suggested that candidates for TKR have "moderate to severe persistent pain not adequately relieved by an extended course of nonsurgical management," but the persistence referred to remained undefined and the only instruments mentioned are the WOMAC, the New Zealand Priority Criteria for Major Joint Replacement, the Knee Society Score, and the Hospital for Special Surgery Knee Score, none of which explicitly evaluates the consistency of the pain at repeated intervals as we have measured here ${ }^{19}$.

We explicitly examined the consistency of subject experience of pain at 2 timepoints separated by a month, and thereby have been able to quantitatively define the persistence of pain. WOMAC pain severity measures are phrased in such a way that they obtain intensity measures during a particular time period; the FKP question is phrased to focus on frequency of the pain across a time period. Although there will certainly be some collinearity between the measures, the dimensions of pain measured are intrinsically very different, and we see in this analysis that they do have effects independent of each other. It is also worth noting that although one might intuitively think that consistency of pain plays a role in predicting TKR, to our knowledge, few if any investigations have tested this hypothesis in a longitudinal epidemiologic study. We believe that when new guidelines for TKR are developed, quantitatively-defined pain consistency should be considered as one indication for TKR.

The strengths of our study include a well designed and executed prospective cohort study, a very high rate of followup over the 30 months, and an outcome where most of the TKR were confirmed by radiographs or medical documentation. We were able to control for a wide variety of potential confounders because of the availability of extensive information collected at baseline in MOST. We have also been able to address significant concerns about confounding and collinearity between consistency and severity of pain measures. Our results demonstrate an excellent, albeit not perfect, dose-response effect for consistency of pain as a predictor of TKR. Lastly, our results have face validity and for the first time establish that an intuitively plausible relation between consistency of pain and risk of TKR exists.

Our study has limitations. First, the dates that TKR were performed were not available, and so we were unable to perform a survival analysis, which might have improved our understanding of the timeframe of the effect of our consistency measure. Second, it is possible that despite our attempts to control for confounding, psychological state and other factors remained as residual confounders. There also may remain some collinearity of the WOMAC measure and the consistency measure not accounted for completely by our analysis method. Further, establishing consistency using only 2 timepoints likely does not identify the full range of potential levels of consistency and thus some misclassification is likely.

We found that consistency of pain in knee OA is strongly associated with TKR, independent of other known risk factors, including pain severity. Consistency of pain may represent an important new tool and add additional information to predict who will ultimately require TKR, and should be considered for inclusion in future guidelines for performing TKR.

\section{ACKNOWLEDGMENT}

We thank the participants and staff in the Multicenter Osteoarthritis (MOST) Study.

\section{REFERENCES}

1. Lawrence RC, Felson DT, Helmick CG, Arnold LM, Choi H, Deyo RA, et al. Estimates of the prevalence of arthritis and other rheumatic conditions in the United States. Part II. Arthritis Rheum 2008;58:26-35.

2. Klein RJ, Schoenborn CA. Age adjustment using the 2000 projected U.S. population. Healthy People 2010 Stat Notes 2001;20:1-10

3. Flegal KM, Carroll MD, Ogden CL, Curtin LR. Prevalence and trends in obesity among US adults, 1999-2008. JAMA 2010;303:235-41.

4. Flegal KM, Carroll MD, Ogden CL, Johnson CL. Prevalence and trends in obesity among US adults, 1999-2000. JAMA 2002;288:1723-7

5. Ogden CL, Carroll MD, Curtin LR, McDowell MA, Tabak CJ, Flegal KM. Prevalence of overweight and obesity in the United States, 1999-2004. JAMA 2006;295:1549-55.

6. Kim S. Changes in surgical loads and economic burden of hip and knee replacements in the US: 1997-2004. Arthritis Rheum 2008;59:481-8.

7. Health, United States, 2009: With Special Feature on Medical Technology. Hyattsville, MD: National Center for Health Statistics; 2010.

8. Hill CL, Gale DG, Chaisson CE, Skinner K, Kazis L, Gale ME, et al. Knee effusions, popliteal cysts, and synovial thickening: association with knee pain in osteoarthritis. J Rheumatol 2001;28:1330-7.

9. Hutchings A, Calloway M, Choy E, Hooper M, Hunter DJ, Jordan 
JM, et al. The Longitudinal Examination of Arthritis Pain (LEAP) study: relationships between weekly fluctuations in patient-rated joint pain and other health outcomes. J Rheumatol 2007; 34:2291-300.

10. Felson DT, Anderson JJ, Naimark A, Walker AM, Meenan RF. Obesity and knee osteoarthritis. The Framingham Study. Ann Intern Med 1988;109:18-24.

11. Spector TD, Hart DJ, Doyle DV. Incidence and progression of osteoarthritis in women with unilateral knee disease in the general population: the effect of obesity. Ann Rheum Dis 1994;53:565-8.

12. Hawker GA, Guan J, Croxford R, Coyte PC, Glazier RH, Harvey $\mathrm{BJ}$, et al. A prospective population-based study of the predictors of undergoing total joint arthroplasty. Arthritis Rheum 2006; 54:3212-20.

13. Neogi T, Felson D, Niu J, Nevitt M, Lewis CE, Aliabadi P, et al. Association between radiographic features of knee osteoarthritis and pain: results from two cohort studies. BMJ 2009;339:b2844.

14. Felson DT, Niu J, Guermazi A, Roemer F, Aliabadi P, Clancy M, et al. Correlation of the development of knee pain with enlarging bone marrow lesions on magnetic resonance imaging. Arthritis Rheum 2007;56:2986-92.
15. Jain NB, Higgins LD, Ozumba D, Guller U, Cronin M, Pietrobon $\mathrm{R}$, et al. Trends in epidemiology of knee arthroplasty in the United States, 1990-2000. Arthritis Rheum 2005;52:3928-33.

16. Escobar A, Quintana JM, Arostegui I, Azkarate J, Guenaga JI, Arenaza JC, et al. Development of explicit criteria for total knee replacement. Int J Technol Assess Health Care 2003;19:57-70.

17. Quintana JM, Escobar A, Arostegui I, Bilbao A, Azkarate J, Goenaga JI, et al. Health-related quality of life and appropriateness of knee or hip joint replacement. Arch Intern Med 2006;166:220-6.

18. De Coster C, McMillan S, Brant R, McGurran J, Noseworthy T. The Western Canada Waiting List Project: development of a priority referral score for hip and knee arthroplasty. J Eval Clin Pract 2007;13:192-6; quiz 7.

19. NIH Consensus Statement on Total Knee Replacement. NIH Consens State Sci Statements 2003;20:1-32. 